ESAIM: PROCEEDINGS, March 2012, Vol. 35, p. 228-233

Fédération Denis Poisson (Orléans-Tours) et E. Trélat (UPMC), Editors

\title{
HOMOGENIZATION OF A CONDUCTIVE-RADIATIVE HEAT TRANSFER PROBLEM
}

\author{
THE CONTRIBUTION OF A SECOND ORDER CORRECTOR ${ }^{*, * *}$
}

\author{
ZAKARIA HABIBI ${ }^{1}$
}

\begin{abstract}
This paper focuses on the contribution of the second order corrector in periodic homogenization applied to a conductive-radiative heat transfer problem. Especially, for a heat conduction problem in a periodically perforated domain with a non-local boundary condition modelling the radiative heat transfer, if this model contains an oscillating thermal source and a thermal exchange with the perforations, the second order corrector helps us to model the gradients which appear between the source area and the perforations.

Résumé. Ce papier est consacré à montrer l'influence du correcteur de second ordre en homogénéisation périodique. Dans l'homogénéisation d'un problème de conduction rayonnement dans un domaine périodiquement perforé par plusieurs trous, on peut voir une contribution non négligeable de ce correcteur lors de la présence d'une source thermique oscillante et d'un échange thermique dans les perforations. Ce correcteur nous permet de modéliser les gradients qui apparaissent entre la zone de la source thermique et les perforations.
\end{abstract}

\section{INTRODUCTION}

We are interested in the homogenization of a conductive-radiative heat transfer in a domain periodically perforated by several infinitely small holes. Despite the presence of various applications for this model, this study is dedicated to the nuclear reactor industry (see [6]). It is adapted to the so-called gas-cooled reactors which are a promising concept for the 4 th generation reactors. The core of this reactors type is composed by many prismatic blocks of graphite in which are inserted the fuel compacts (here the thermal sources). Each block is periodically traversed by several infinitely small holes where the coolant (Helium) circulates. We suppose here that the holes are disconnected, namely each hole is compactly embedded in its periodicity cell (see [3] for the case of non-isolated cylinders). For our study, the geometry will be scaled down into two states: a fluid state composed by the coolant gas and a solid state composed by the fuel and the graphite. Therefore, any study of heat transfer in such a geometry has to take into account the local difference between these areas. We notice that the total number of holes is very high and their size is very small compared to the size of the core. Consequently, the numerical analysis of such models requires a very fine mesh of this structure. This induces a

\footnotetext{
* The author thanks G. Allaire (gregoire.allaire@polytechnique.fr) and A. Stietel (anne.stietel@cea.fr) for their useful collaboration.

** This work has been supported by the French Atomic Energy and Alternative Energy Commission, DEN/DM2S at CEA Saclay.

1 CEA \& CMAP, zakaria.habibi@polytechnique.edu
}

(C) EDP Sciences, SMAI 2012 

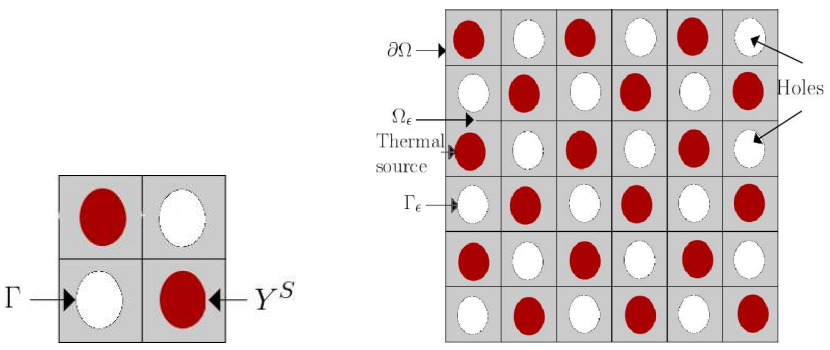

FIGURE 1 . The periodic domain $\Omega_{\epsilon}$ (right) and its reference cell $Y$ (left)

very expensive numerical resolution that becomes impossible for a real geometry of a reactor core. Therefore, our objective is to define a homogeneous model equivalent to those modes of heat transfer that is less expensive, in term of CPU time and memory, and converging to the direct model. Specifically, we will give a clear definition of the homogenized model and its effective parameters. The homogenization of this conductive-radiative heat transfer was already carried out by G. Allaire and K. El Ganaoui in [2]. Thus, the peculiarity of the actual study comes from the problems encountered when we have simultaneously a periodic oscillating thermal source and a condition of exchange along the holes. In this case, gradients of temperature are emerging between the area of the source and the holes. These gradients do not appear in the homogeneous solution corrected only by the corrector of order 1 (see Figure 3). Hence our need to analyse the contribution of the second order corrector since it is the only term that depends on the local values of the thermal source and the heat exchange. We will see later in this paper, that these values are averaged in the homogenized problem (which makes sense since all the parameters of this model are homogenized parameters), and the problem on the first corrector depends only on the radiative condition on the holes walls (see $[8,9]$ for further detail).

\section{Setting of the PRoblem}

\subsection{Geometry}

Let $\Omega$ be a bounded set of $\mathbb{R}^{d}$ such that $\Omega=\prod_{j=1}^{d}\left(0, L_{j}\right)$ (here $d=2$ ). We define a domain $\Omega_{\epsilon}$ as the domain $\Omega$ without a collection of holes $\left(\tau_{\epsilon, i}\right)_{i=1 \ldots N(\epsilon)}$. The domain $\Omega$ is subdivided into $N(\epsilon)$ periodic cells $\left(Y_{\epsilon, i}\right)_{i=1 \ldots N(\epsilon)}$, each of them is equal, up to a translation, to the same unit cell $Y=\prod_{j=1}^{d}\left(0, l_{j}\right)$. We denote by $\Gamma$ the walls of the holes in $Y$ and by $Y^{S}$ the solid part of $Y$. We suppose also that for each $i, \tau_{\epsilon, i} \subset \subset Y_{\epsilon, i}$. In summary we have $\quad \Omega_{\epsilon}=\Omega \backslash\left(\bigcup_{i=1}^{N(\epsilon)} \tau_{\epsilon, i}\right)=\bigcup_{i=1}^{N(\epsilon)} Y_{\epsilon, i}^{S}, \quad \Gamma_{\epsilon}=\bigcup_{i=1}^{N(\epsilon)} \partial \tau_{\epsilon, i}=\bigcup_{i=1}^{N(\epsilon)} \Gamma_{\epsilon, i}$ where $Y_{\epsilon, i}^{S}=Y_{\epsilon, i} \tau_{\epsilon, i}$ and $\Gamma_{\epsilon, i}=\partial \tau_{\epsilon, i}$.

\subsection{Governing equations}

Eventually, the governing equations of our model are

$$
\left\{\begin{aligned}
-\operatorname{div}\left(K_{\epsilon} \nabla T_{\epsilon}\right) & =f_{\epsilon} & & \text { in } \Omega_{\epsilon}, \\
-K_{\epsilon} \nabla T_{\epsilon} \cdot n & =\epsilon h_{\epsilon}\left(T_{\epsilon}-T_{\text {gas }}\right)+\frac{\sigma}{\epsilon} G_{\epsilon}\left(T_{\epsilon}\right) & & \text { on } \Gamma_{\epsilon}, \\
T_{\epsilon} & =g & & \text { on } \partial \Omega,
\end{aligned}\right.
$$

where $K_{\epsilon}(x)=K\left(x, \frac{x}{\epsilon}\right)$ and $K$ is the conductivity tensor of the unit cell $Y$. We assume $K$ to be symmetric, uniformly coercive and bounded in norm $\mathrm{L}^{\infty} . h_{\epsilon}(x)=h\left(x, \frac{x}{\epsilon}\right)$ is the exchange coefficient in the hole walls, $n$ is the unit outward normal on $\Gamma_{\epsilon}, f_{\epsilon}(x)=f\left(x, \frac{x}{\epsilon}\right)$ is the oscillating thermal source where $f(x, y) \in L^{2}\left(\Omega, L^{2}(Y)\right)$ 
and $f \geq 0, T_{\text {gas }}(x)$ is the temperature in the holes (supposed known). $\sigma$ is the Stefan-Boltzmann constant and $G_{\epsilon}$ is the radiative operator given by

$$
G_{\epsilon}\left(T_{\epsilon}\right)=e\left(I d-\zeta_{\epsilon}\right)\left(I d-(1-e) \zeta_{\epsilon}\right)^{-1}\left(T_{\epsilon}^{4}\right) \quad \text { with } \zeta_{\epsilon}(f)(s)=\int_{\Gamma_{\epsilon, i}} F(s, x) f(x) d x .
$$

where $F$ is the view factor (see [10]) and $e$ is the emissivity of the holes walls.

\section{Homogenization By ASYMPtotic EXPANSION}

The homogenized problem can be obtained heuristically by the method of two-scale asymptotic expansion [4]. The starting point of this method is to assume that the solution $T_{\epsilon}$ of problem (1) is given by the series

$$
T_{\epsilon}=T_{0}(x)+\epsilon T_{1}\left(x, \frac{x}{\epsilon}\right)+\epsilon^{2} T_{2}\left(x, \frac{x}{\epsilon}\right)+\ldots
$$

Our main result is the following:

Proposition 2.1. (proof in [8,9]) Under ansatz (2), we show that $T_{0}$ is the solution of the homogenized problem

$$
\left\{\begin{aligned}
-\operatorname{div}\left(K^{*}(x) \nabla T_{0}(x)\right)+h^{*}(x)\left(T_{0}(x)-T_{\text {gas }}(x)\right) & =f^{*}(x) & & \text { in } \Omega \\
T_{0}(x) & =0 & & \text { on } \partial \Omega
\end{aligned}\right.
$$

where the homogenized conductivity tensor $K^{*}$ is given by its entries, for $j, k=1,2$,

$$
K_{j, k}^{*}=\frac{1}{|Y|}\left[\int_{Y^{S}} K(x, y)\left(e_{j}+\nabla_{y} \omega_{j}(y)\right) \cdot\left(e_{k}+\nabla_{y} \omega_{k}(y)\right) d y+4 \sigma T_{0}^{3} \int_{\Gamma} G\left(\omega_{k}(y)+y_{k}\right)\left(\omega_{j}(y)+y_{j}\right) d y\right]
$$

with $G \equiv G_{\epsilon}$ in $\Gamma$. The homogenized source $f^{*}$ is given by $f^{*}(x)=\frac{1}{|Y|} \int_{Y^{S}} f(x, y) d y$. The homogenized exchange coefficient $h^{*}$ is given by $h^{*}(x)=\frac{1}{|Y|} \int_{Y_{S}} h(x, y) d y$, and $\left(\omega_{k}(x, y)\right)_{1 \leq k \leq 2}$ are the solutions of the cell problems

$$
\left\{\begin{aligned}
-\operatorname{div}_{y} K(x, y)\left(e_{j}+\nabla_{y} \omega_{j}\right) & =0 & & \text { in } Y^{S} \\
K(x, y)\left(e_{j}+\nabla_{y} \omega_{j}\right) \cdot n & =4 \sigma T_{0}^{3} G\left(\omega_{j}(y)+y_{j}\right) & & \text { on } \Gamma \\
y & \mapsto \omega(y) & & \text { is } Y \text {-periodic }
\end{aligned}\right.
$$

Furthermore, the first corrector $T_{1}(x, y)$ is given by $T_{1}(x, y)=\sum_{j=1}^{2} \frac{\partial T_{0}}{\partial x_{j}}(x) \omega_{j}(x, y)+\tilde{T}_{1}(x)$,

and the second one $T_{2}(x, y)$ is the solution of the cell problem

$$
\begin{cases}-\operatorname{div}_{y}\left(K\left[\nabla_{y} T_{2}+\nabla_{x} T_{1}\right]\right)=\operatorname{div}_{x}\left(K\left[\nabla_{x} T_{0}+\nabla_{y} T_{1}\right]\right)+f & \text { in } Y^{S} \\ -K\left[\nabla_{y} T_{2}+\nabla_{x} T_{1}\right] \cdot n=h\left(T_{0}-T_{g a s}\right)+4 \sigma T_{0}^{3}\left[G\left(T_{2}+\nabla_{x} T_{1} \cdot y+\nabla \nabla T_{0} y \cdot y\right)-G\left(\nabla_{x} \nabla_{x} T_{0} y+\nabla_{x} T_{1}\right) \cdot y\right] & \text { on } \Gamma \\ T_{2} \text { is } Y \text {-periodic. } & \end{cases}
$$

where all the functions are evaluated at $(x, y) \in \Omega \times Y^{S}$ except $T_{0}$ and $T_{\text {gas }}$ which are evaluated at $x \in \Omega$. 
Proposition 2.2. If we suppose, in Proposition 2.1, that the functions $f$ and $h$ verify $f(x, y)=F(x) f_{\#}(y)$ and $h(x, y)=H(x) h_{\#}(y)$, and that the conductivity tensor $K_{\epsilon}$ is given by $K_{\epsilon}(x)=K\left(\frac{x}{\epsilon}\right)$ instead of $K_{\epsilon}(x)=K\left(x, \frac{x}{\epsilon}\right)$, we have:

$$
\begin{aligned}
& f^{*}(x)=\frac{1}{|Y|} \int_{Y^{S}} f(x, y) d y=\frac{F(x)}{|Y|} \int_{Y^{S}} f_{\#}(y) d y=F(x) F^{*}, \\
& h^{*}(x)=\frac{1}{|Y|} \int_{Y^{S}} h(x, y) d y=\frac{H(x)}{|Y|} \int_{Y^{S}} h_{\#}(y) d y=H(x) H^{*},
\end{aligned}
$$

and $T_{2}(x, y)$ is given by:

$$
T_{2}(x, y)=\left(T_{2}^{1}(y)+T_{2}^{2}(y)\right) F(x)+T_{2}^{3}(y) H(x)\left(T_{0}(x)-T_{g a s}(x)\right)+\sum_{i, j=1}^{2} \frac{\partial^{2} T_{0}}{\partial x_{i} \partial x_{j}}(x) \theta_{i, j}(y)+\tilde{T}_{2}(x)
$$

where $T_{2}^{1}, T_{2}^{2}, T_{2}^{3}$ and $\left(\theta_{i, j}\right)_{i, j=1,2}$ are the solutions of the cell problems:

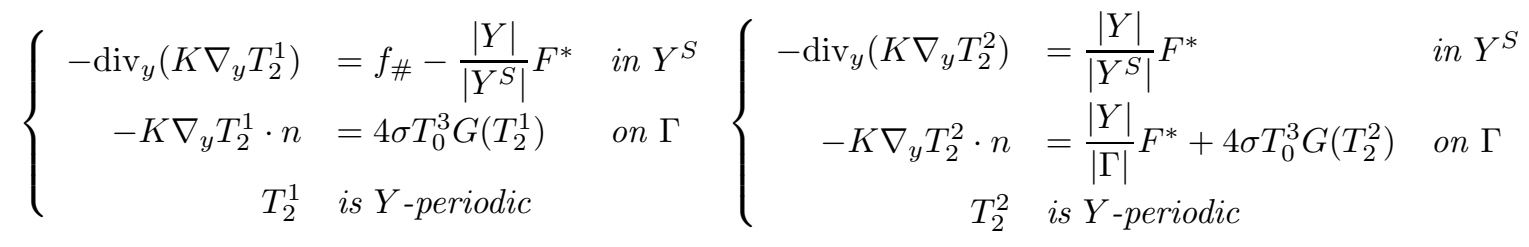

$$
\begin{aligned}
& \left\{\begin{array}{rlr}
-\operatorname{div}_{y}\left(K \nabla_{y} T_{2}^{3}\right) & =0 & \text { in } Y^{S} \\
-K \nabla_{y} T_{2}^{3}(y) \cdot n & =\left(h_{\#}-\frac{|Y|}{|\Gamma|} H^{*}\right)+4 \sigma T_{0}^{3} G\left(T_{2}^{3}\right) & \text { on } \Gamma \\
T_{2}^{3} & \text { is } Y \text {-periodic } &
\end{array}\right. \\
& \left\{\begin{array}{rlr}
-\operatorname{div}_{y}\left(K \nabla_{y} \theta_{i, j}\right) & \left.\left.=\operatorname{div}_{y}\left(K e_{j} \omega_{i}\right)+K_{i, j}+K^{t} e_{j} \cdot \nabla_{y} \omega_{i}\right)\right] & \text { in } Y^{S} \\
-K \nabla_{y} \theta_{i, j} \cdot n & =K e_{j} \omega_{i}+\frac{|Y|}{|\Gamma|} K_{i, j}^{*}+4 \sigma T_{0}^{3} y_{j} G\left(\omega_{i}+y_{i}\right)+4 \sigma T_{0}^{3} G\left(\theta_{i, j}+y_{j} \omega_{i}+\frac{1}{2} y_{i} y_{j}\right) & \text { on } \Gamma \\
\theta_{i, j} & \text { is Y-periodic } &
\end{array}\right.
\end{aligned}
$$

Remark 2.3. (1) The non-oscillating functions $\tilde{T}_{1}$ and $\tilde{T}_{2}$ respectively in (3) and (4) are undetermined. We can see, in [4], that if we stop the expansion 2 at order 1 , the function $\tilde{T}_{1}$ (and a fortiori $\tilde{T}_{2}$ ) plays no role in our approximation and we can set it to zero. However, if we are interested in correctors $T_{i}$, with $i>1$, this function should satisfy an additional compatibility condition (see [4]).

(2) In the cell problems on $T_{1}$ and $T_{2}$, the macroscopic variable $x$ plays only the role of a parameter.

(3) In the cell problem on $T_{2}$, we remark that $T_{2}$ depends on the local values of the thermal source $f(x, y)$ and the thermal exchange coefficient $h(x, y)$ unlike the homogenized problem which depends on their averages in $Y^{S}$. Since the cell problem on $T_{1}$ depends only on the diffusivity tensor $K$ and the radiative operator $G$, we take a particular interest in the second corrector as it is the first term of (2), which contains the local variations between the thermal source and the heat exchange.

\section{NumericAl RESUlts}

In this section we describe some numerical experiments to study the asymptotic behaviour of the non-linear heat transfer model (1). Our goal is to show the efficiency of our proposed homogenization procedure, to validate 
it by comparing the reconstructed solution of the homogenized model with the numerical solution of the exact model (1) for smaller and smaller values of $\epsilon$ and to exhibit a numerical rate of convergence in terms of $\epsilon$. All computations have been done with the finite element code CAST3M [5] developed at the French Atomic and Alternative Energy Commission (CEA). We now give our computational parameters for a reference 2D computation corresponding to $\epsilon=\frac{1}{4}$. The geometry corresponds to a cross-section of a typical fuel assembly for a gas-cooled nuclear reactor (see [7] for further references). The domain is $\prod_{j=1}^{2}\left(0, L_{j}\right)$, with, $L_{1}=0.48 \mathrm{~m}$ and $L_{2}=1.12 m$. Each periodicity cell is equal to $\prod_{j=1}^{2}\left(0, \epsilon L_{j}\right)$, each one contains 2 hollow cylinders (holes) (see Figure 1), the radius of which is equal to $\epsilon 0.014 m$. The emissivity of the holes boundaries is equal to $e=1$. The oscillating thermal source $f$ is equal to $f=6 M W$ in disks strictly included in $\Omega_{\epsilon}$ (with the same size as the fluids disks: channels) such that we have a disc between each two fluid holes (see Figure 1). The source is set to zero elsewhere. We enforce periodic boundary conditions in the $x_{1}$ direction and Dirichlet boundary conditions in the other directions which are given by $T_{\epsilon}(x)=800 \mathrm{~K}$ on the boundaries corresponding to $x_{2}=0$ and $T_{\epsilon}(x)=1200 \mathrm{~K}$ on the boundaries corresponding to $x_{3}=L_{3}$. $\tilde{T}_{1}$ and $\tilde{T}_{2}$ are set to zero. The physical value of the isotropic conductivity is $30 \mathrm{Wm}^{-1} \mathrm{~K}^{-1}$. To avoid an excessive computational burden, we have chosen periodic boundary condition in the $x_{1}$ direction which implies that it is not necessary to decrease the cell size in the $x_{1}$ direction. Therefore, $\ell_{1}=1 / 3 L_{1}$ is fixed and we simply add cells in the $x_{2}$ direction, decreasing $\epsilon$ from $1 / 4$ to $1 / 10$ with a unit step. In Figure 2 we plot the direct, homogenized and reconstructed solutions

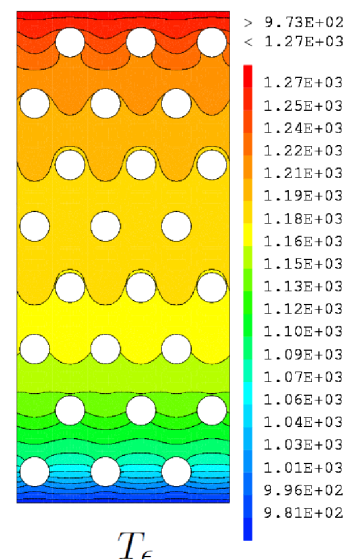

$T_{\epsilon}$

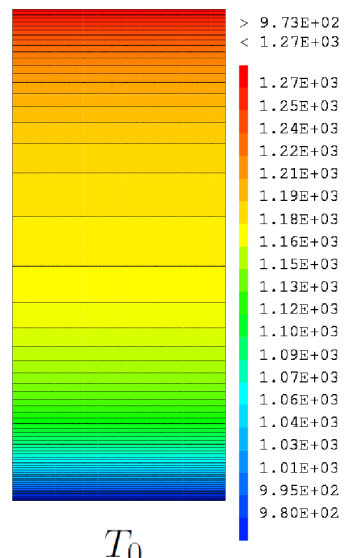

$T_{0}$

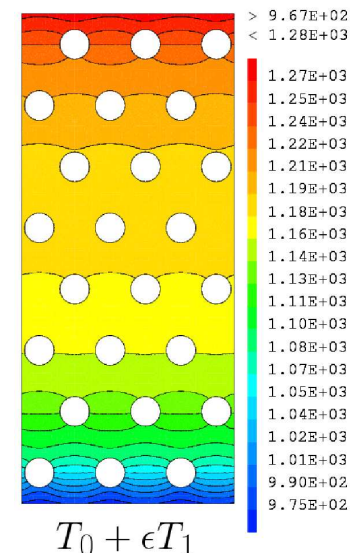

$T_{0}+\epsilon T_{1}$

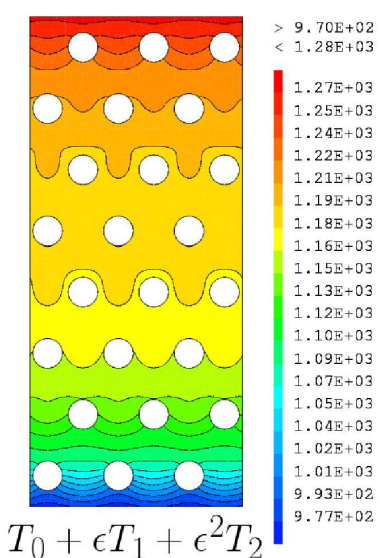

Figure 2. Solutions in $\Omega_{\epsilon}$ for $\epsilon=1 / 4$

computed for a value of $\epsilon=\epsilon_{0}=1 / 4$. We plot also in Figure 2 these solution in the segment defined by $p 1=[a 1, a 2]$ where $a 1=(1.75 E-028.25 E-02)$ and $a 2=(8.72 E-0217.6 E-02)$.

In $[3,9]$, we provide a rigorous mathematical justification of the homogenization process by using the method of two-scale convergence $[1,11]$. This method helps us to justify the two first terms of $(2)$. To show the convergence of our method when it includes the second order corrector $\epsilon^{2} T_{2}$, we draw, in Figure (4), the relative error related to temperature in the domain $\Omega_{\epsilon}$ with periodic boundary conditions in both directions $x_{1}$ and $x_{2}$ to avoid complications with boundary layers. We compare this error with the period $\epsilon$. The slopes shows that the relative error $\operatorname{ERR}(T)$ behaves like $\epsilon^{2}$ which is in accordance with that theoretically predicted for a pure diffusion problem in [4]. We notice also that, in absence of the boundary layers, the relative error related to the correction by only the first order corrector $T_{1}$ behaves also like $\epsilon^{2}$ but remains, for each $\epsilon$, larger than $\operatorname{ERR}(T)$. This means that adding the second order corrector $T_{2}$ does not improve significantly the convergence order of our method. However, for a fixed $\epsilon$ (often by industrial constraints like the hole size in the present application), this corrector is very useful, even essential, to have a good approximation by homogenization of the heat transfer problem (1). 


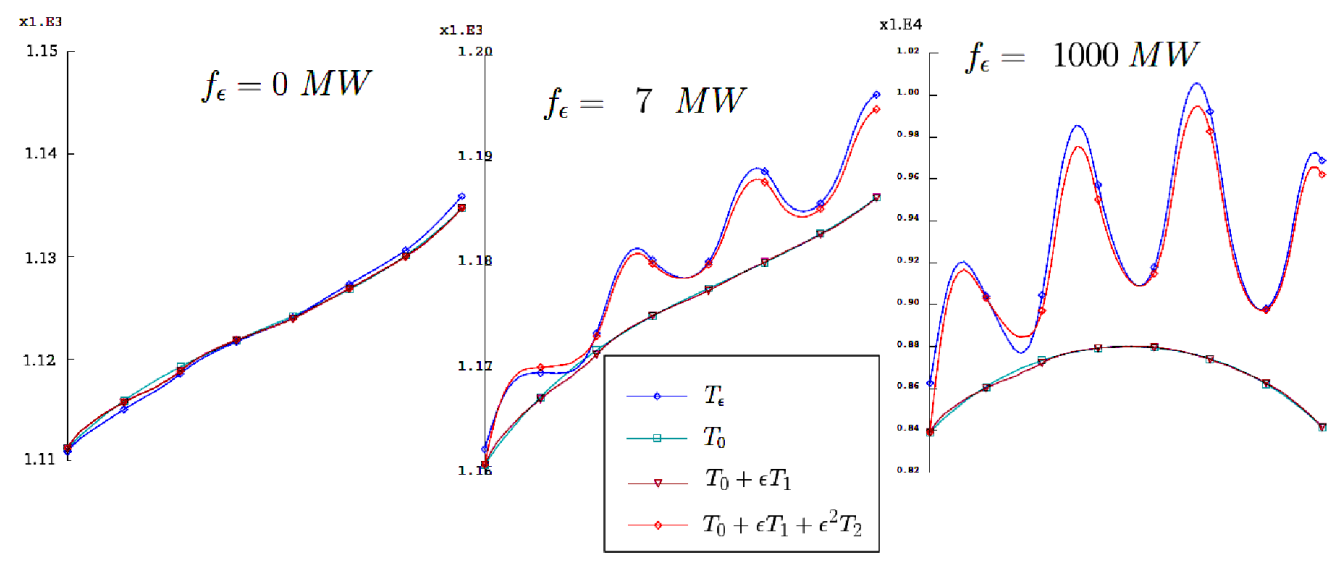

Figure 3. Solutions in the segment $p 1$ for $\epsilon=1 / 4$ and $f_{\epsilon}=0 M W$ (left), $f_{\epsilon}=7 M W$ (center), $f_{\epsilon}=1000 M W$ (right)

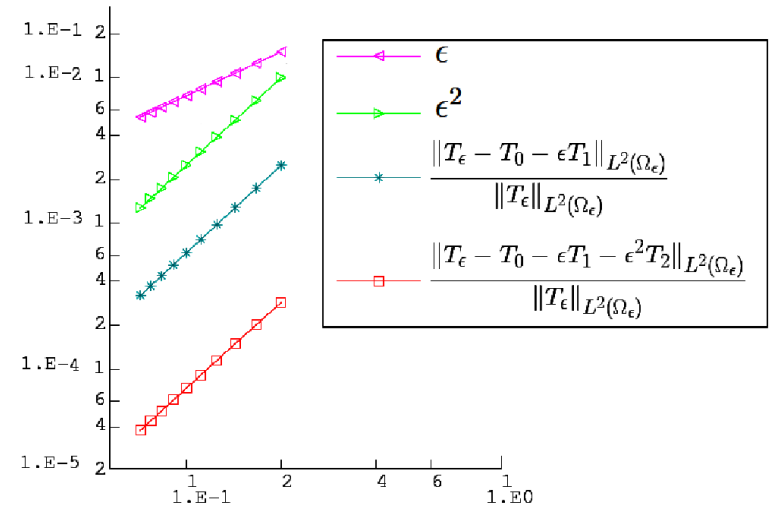

FIGURE 4. Relatives errors on the temperature

\section{REFERENCES}

[1] G. Allaire. Homogenization and two-scale convergence. SIAM J. Math. Anal., 23(6):1482-1518, 1992.

[2] G. Allaire and K. El Ganaoui. Homogenization of a conductive and radiative heat transfer problem. Multiscale Model. Simul., $7(3): 1148-1170,2008$.

[3] G. Allaire and Z. Habibi. Homogenization of a conductive, convective and radiative heat transfer problem. To appear.

[4] A. Bensoussan, J. L. Lions, and G. Papanicolaou. Asymptotic analysis for periodic structures, volume 5 of Studies in Mathematics and its Applications. North-Holland Publishing Co., Amsterdam, 1978.

[5] Cast3M. http://www-cast3m.cea.fr/cast3m/index.jsp.

[6] CEA e-den. Les réacteurs nucléaires à caloporteur gaz. CEA Saclay et Le Moniteur Editions. Monographie Den,2006. http://nucleaire.cea.fr/fr/publications/pdf/M0-fr.pdf.

[7] K. El Ganaoui. Homogénéisation de modèles de transferts thermiques et radiatifs : application au coeur des réacteurs à caloporteur gaz. PhD thesis, Ecole Polytechnique, 2006.

[8] Z. Habibi. Homogenization of a conductive-radiative heat transfer problem, the contribution of a second order corrector. ESAIM proc. (submitted).

[9] Z. Habibi. Homogénéisation et convergence à deux échelles lors d'échanges thermiques stationnaires et transitoires dans un coeur de réacteur à caloporteur gaz. PhD thesis, Ecole Polytechnique, 2011.

[10] F. M. Modest. Radiative heat transfer. Academic Press, 2 edition, 2003.

[11] G. Nguetseng. A general convergence result for a functional related to the theory of homogenization. SIAM J. Math. Anal., 20(3):608-623, 1989. 